\title{
Preparation, Electrical Conduction, Hall Effect and Photoconductivity of Thallium Monosulfide Single Crystal
}

\author{
H. A. Elshaikh, I. M. Ashraf and A. M. Badr \\ Physics Department, Faculty of Science (Aswan), South Valley \\ University, Egypt.
}

Thallium monosulfide single crystal was prepared and characterized by $x$-ray diffraction. The electrical measurements in the temperature range form 100 to $360 \mathrm{~K}$, show that $\sigma_{\perp}=8.9 \times 10^{-7}(\Omega . \mathrm{cm})^{-1}$ when the current flow direction makes right angle to the cleavage plane of the crystals, and $\sigma_{/ /}=1.39$ $\times 10^{-7}(\Omega . c m)^{-1}$ when the current flows is parallel to the cleavage plane. The width of the band gap was calculated from the electrical data and found to be $E_{g}=1.1 \mathrm{eV}$. The anisotropy of the electrical conductivity $\left(\sigma_{\perp} / \sigma_{/ /}\right)$was also studied in this work. The photosensitivity was calculated for different levels of illumination which showed that the recombination process in TIS single crystals is monomolecular.

\section{Introduction:}

Thallium chalcogenides received a great deal of attention due to their optical and electrical properties in view of the possible optoelectronic device applications [1-4]. Semiconductors with the general formula $\mathrm{R} 1^{\mathrm{I}} \mathrm{M}^{\mathrm{III}} \mathrm{X}_{2}(\mathrm{M}=\mathrm{In}$ or $\mathrm{Ga}$ and $\mathrm{X}=\mathrm{S}$, Se or Te) have three structure types: type I, tetragonal TlSe structure [5]; type II, monoclinic $\mathrm{TlGaSe}_{2}$ structure [6]; type III, tetragonal layer-type structure [7]. Kashida and Nakamura [8] reported that TIS is a mixed valence compound and is classified in this family, as represented by the formula $\mathrm{Tl}^{\mathrm{I}} \mathrm{Tl}^{\mathrm{III}} \mathrm{S}_{2}$. They also showed that two different structures with tetragonal symmetry are identified for TIS. The first with space group $14 / \mathrm{mcm}$ having a lattice parameters of: $\mathrm{a}=\mathrm{b}=7.785(2) \AA, \mathrm{c}=6.802(2) \AA$ and $\mathrm{Z}=8$ (the TlSe type). Chains formed of edge-connected TIIIIS4 tetrahedra, characterize this structure. Monovalent $\mathrm{Tl}^{1}$ ions are located between the chains and have eightfold antiprismatic coordination of the sulfur ions. The second structure has a space group $\mathrm{P} 4{ }_{1} 2{ }_{1} 2$ : $\mathrm{a}=\mathrm{b}=7.803(9) \AA \hat{A}, \mathrm{c}=29.55(2) \AA \hat{~}$ and $\mathrm{Z}=32$. Layers formed of corner-connected TIIIIS4 tetrahedra characterize the structure. Monovalent 
$\mathrm{Tl}^{1}$ ions are located between the layers and have sevenfold capped trigonal coordination of the sulfur ions. Recent $x$-ray studies have shown that TIS crystallizes in both the TlSe-type and the $\mathrm{TlGaSe}_{2}$-type structures [7-9]. The $\mathrm{TlGaSe}_{2}$-type (layer) of TlS crystal undergoes successive ferroelectric phase transitions [9]. The microscopic origin of the phase transitions is the small displacements of $\mathrm{Tl}^{1+}$ ions relative to S ions (see Fig. 1). Although TlS is known as a good photoconductor, its electronic structure is not well known. In an earlier $\mathrm{x}$-ray photoemission study of the TISe-type (chain) TIS, it was reported that no splitting of the $5 \mathrm{~d}$ lines was observed for the two $\mathrm{Tl}$ ions having different valences [10]. Similar results were reported in an $\mathrm{x}$-ray photoemission study of $\mathrm{Pb}_{3} \mathrm{O}_{4}$, which contains divalent and tetravalent $\mathrm{Pb}$ ions [11]. Using a synchrotron radiation of TIS crystals with both the TlSe-and $\mathrm{TlGaSe}_{2}$-type structures, photoemission studies have been reported [12]. It was reported in this study that the line shape of the $\mathrm{Tl} 5 \mathrm{~d}$ core level is more asymmetric for the TlSe-type than for the $\mathrm{TlGaSe}_{2}$-type. A chemical method for depositing thin films of TIS onto glass substrates has been developed using a thallous nitrate solution, ammonia and thiourea as the reacting agents [13]. The films were found to be p-type with an optical energy gap of about $1.02 \mathrm{eV}$. An investigation of the dielectric property of monoclinic thallium monosulfide has been reported in the temperature range from 285 to $380 \mathrm{~K}$ [14]. It was found that the dielectric constant show anomalous increase at 318.6 and $341.1 \mathrm{~K}$ suggesting the occurrence of successive phase transitions. The electrical properties and infrared spectra of TIS have been also reported by several authors $[15,16]$.

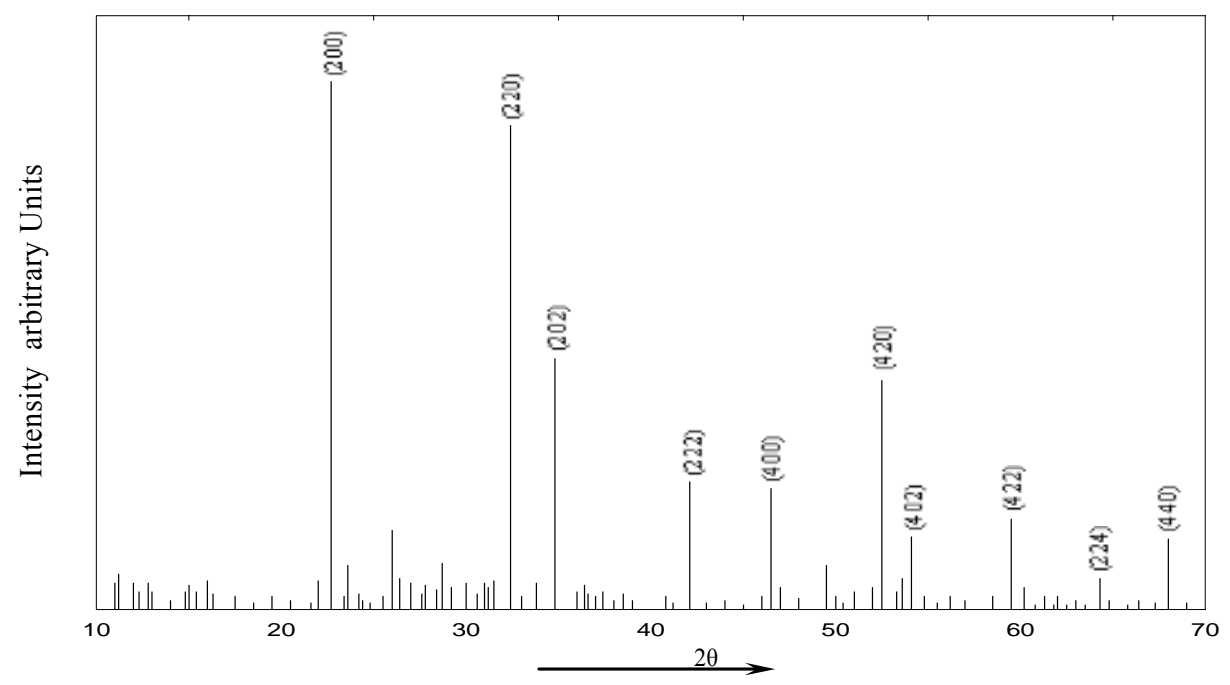

Fig. (1): The powdered XRD pattern of the as-grown TlS crystal at room temperature. 
In the present work, a new homemade technique is used for preparing thallium monosulfide single crystal. The aim of the work is to elucidate the preparation process, anisotropy of the electrical conductivity and photoconductivity for the obtained crystals.

\section{Samples Preparation and the experimental arranged:}

The thallium monosulfide single crystal were prepared by a locally designed apparatus [17]. TIS crystals were prepared from pure (5N) elements (Aldrich) in an evacuated silica tube $\left(10^{-6}\right.$ Torr) of $1.5 \mathrm{~cm}$ in diameter and 20 $\mathrm{cm}$ in length. The charge elements are $3.3176 \mathrm{gm}$ of thallium and $0.5205 \mathrm{gm}$ of sulfur weighted by using an electric balance (Sartorius) with sensitivity of $10^{-4}$ g. The silica tube containing TIS compound was placed in the center of the uniform zone within the work-tube. The temperature in the uniform zone of the work-tube was adjusted by using the digital temperature controllers to be higher than the melting point of TIS compound. This condition was dept for 24 hours, during which the melt was shaken several times in order to accelerate the diffusion of the constituents through each other leading to the melt homogenization. Thereafter the three digital temperature controllers were used to supply a narrow temperature gradient to the length of the uniform zone in which the silica tube containing the compound was placed. Then the crystallization process was achieved by cooling the melt slowly with a rate of 5 ${ }^{\circ} \mathrm{C} / \mathrm{h}$. The crystal structure of the obtained TlS crystal was identified at room temperature. The diffraction pattern of the obtained crystals is compared with the "International Center for Diffraction Data" (ICDD) card. The natures of the obtained crystals have been confirmed by comparing the values of the interplaner distance (d) calculated from the peaks and those recorded in the ICDD card [18] (see Fig. 1 and Table 1). This comparison showed that the resulting TIS compound is a single crystal with tetragonal symmetry, space group: $14 / \mathrm{mcm}$ and lattice parameters: $\mathrm{a}=7.79 \AA, \mathrm{c}=6.79 \AA$ and $\mathrm{Z}=8$. It is evident that the crystal has a high degree of crystallinity indicating that the preparation technique is fairly reliable and satisfactory.

Table (1): The crystallographic data "obtained and standard [17]" for TIS crystals

\begin{tabular}{|c|c|c|c|c|c|c|c|}
\hline \multirow{2}{*}{$\mathrm{h} \mathrm{k} \ell$} & \multirow{2}{*}{$\mathrm{I} / \mathrm{I}_{\mathrm{o}}$} & \multicolumn{2}{|c|}{$\mathrm{d}(\AA)$} & \multirow{2}{*}{$\mathrm{h} \mathrm{k} \ell$} & \multirow{2}{*}{$\mathrm{I} / \mathrm{I}_{\mathrm{o}}$} & \multicolumn{2}{|c|}{$\mathrm{d}(\AA)$} \\
\hline & & Obtained & Standard & & & Obtained & Standard \\
\hline$\left(\begin{array}{lll}2 & 0 & 0\end{array}\right)$ & 60 & 3.91 & 3.893 & $\left(\begin{array}{lll}4 & 2 & 0\end{array}\right)$ & 60 & 1.74 & 1.745 \\
\hline$\left(\begin{array}{lll}2 & 2 & 0\end{array}\right)$ & 60 & 2.76 & 2.746 & $\left.\begin{array}{lll}4 & 0 & 2\end{array}\right)$ & 60 & 1.69 & 1.691 \\
\hline $\begin{array}{llll}\left(\begin{array}{lll}2 & 0 & 2\end{array}\right)\end{array}$ & 80 & 2.57 & 2.557 & $\left(\begin{array}{lll}4 & 2 & 2\end{array}\right)$ & 100 & 1.55 & 1.55 \\
\hline$\left(\begin{array}{lll}2 & 2 & 2\end{array}\right)$ & 60 & 2.14 & 2.14 & $\left(\begin{array}{lll}2 & 2 & 4\end{array}\right)$ & 45 & 1.45 & 1.446 \\
\hline$\left(\begin{array}{lll}4 & 0 & 0\end{array}\right)$ & 40 & 1.95 & 1.946 & $\left(\begin{array}{lll}4 & 4 & 0\end{array}\right)$ & 40 & 1.37 & 1.374 \\
\hline
\end{tabular}


The bulk sample, which is used in the measurements, was prepared by splitting the crystal along he cleavage plane and hence the resultant surface was mirror-like without any mechanical treatment. Then the sample was mounted on the cold finger inside a cryostat (Oxford DN1704-type), which was evacuated to about $10^{-4}$ Torr. The temperature inside the cryostat was controlled by a digital temperature controller (Oxford ITC601-type). The contacts between the samples and the metal electrodes were made by using silver paste. The electrical contacts have been tested by analyzing their $\mathrm{I}-\mathrm{V}$ characteristics and are found to be ohmic in the whole investigated range of the applied voltage. The electrical conductivity measurements were made with an electrometer (Keithly $610 \mathrm{C}$ ). In photoconductivity measurements, excitation was done by a tungsten-filament lamp of 1000 Watt. The incident light was focused by using an optical system consisting of two convex lenses, which enables a homogeneous illumination to be obtained.

\section{Results and Discussions:}

\subsection{Electrical conductivity and Hall effect:}

For electrical conductivity measurements, a sample of (T1S) single crystal was prepared by the cleavage parallel in direction to the layers of these crystals. The resultant plate-like sample was treated to be in a rectangular shape of dimensions $4 \times 1.4 \times 0.8 \mathrm{~mm}$. The electrical conductivity $(\sigma)$ was measured in two cases: one of them is that when the current flow direction makes right angle to the cleavage plane $\left(\sigma_{\perp}\right)$, and the second is when the current flows parallel to the cleavage plane $\left(\sigma_{/ /}\right)$. At room temperature, it was found that $\sigma_{\perp}=$ $8.9 \times 10^{-7}$ and $\sigma_{/ /}=1.39 \times 10^{-6}(\Omega . \mathrm{cm})^{-1}$. The temperature dependence of the electrical conductivity $\left(\sigma_{/ /}\right.$and $\left.\sigma_{\perp}\right)$ for thallium monosulfide single crystal in the whole investigated temperature range (from 100 to $360 \mathrm{~K}$ ) is presented in Fig. (2). It is clear from the figure that the Arrhenius behavior is observed in three temperature regions in which $\sigma_{\perp}$ differs from $\sigma_{/ /}$in their values suggesting anisotropy of the electrical conductivity of TIS single crystal. The first region (I) corresponds to low temperatures, where the electrical conductivity in this range could be attributed to the extrinsic transitions; the second region (II) may be considered as the transient region and conduction occurs via extrinsic regime; the third region (III) corresponds to high temperatures, where the intrinsic transitions are responsible for the current flow. Figure (2) also suggests that the electrical conductivity of TIS single crystal slowly increases in the extrinsic region with increasing temperature satisfying the relation:

$$
\sigma=\sigma_{\mathrm{o}} \exp \left[\frac{-E_{a}}{2 k T}\right]
$$


where $\sigma_{0}$ is the pre-exponential factor and $E_{a}$ is the activation energy. The extrinsic region (I) starts form 140 to $185 \mathrm{~K}$ with activation energy of $0.031 \mathrm{eV}$ for $\sigma_{\perp}$ and from 140 to $175 \mathrm{~K}$ with activation energy of $0.076 \mathrm{eV}$ for $\sigma_{/ /}$. The temperature dependence of electrical conductivity in the transient region is greater than that in the extrinsic region. This region starts from 185 to $200 \mathrm{~K}$ with activation energy of $0.27 \mathrm{eV}$ for $\sigma_{\perp}$ and from 175 to $190 \mathrm{~K}$ with activation energy of $0.24 \mathrm{eV}$ for $\sigma_{/ /}$. The electrical conductivity of TIS single crystal rapidly increases in the intrinsic region with increasing temperature according to following relation, (Fig. 2).

$$
\sigma=\sigma_{\mathrm{o}} \exp \left[\frac{-E g}{2 k T}\right]
$$

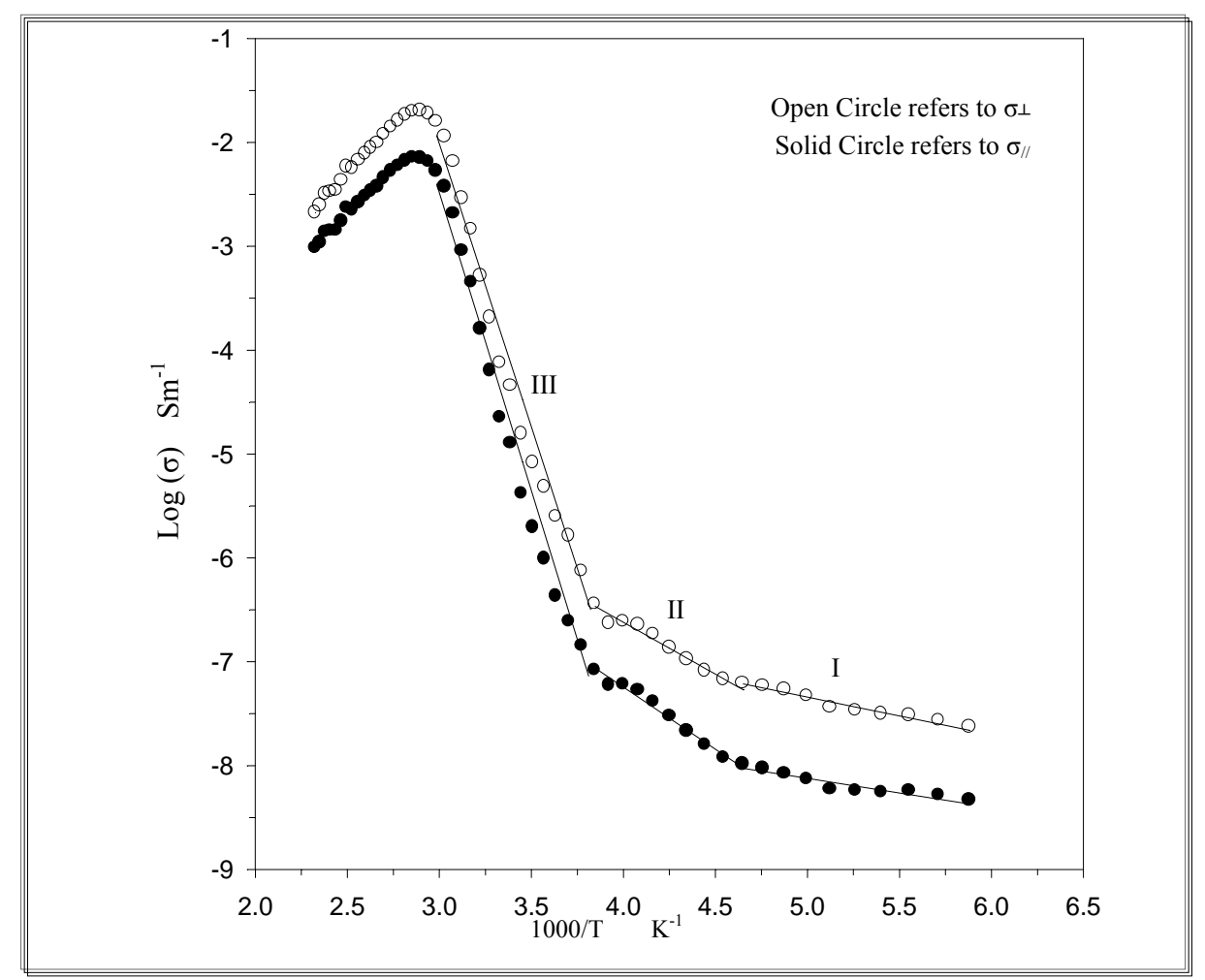

Fig. (2): Reciprocal temperature dependence of electrical conductivity of TIS single crystal

This region starts from 200 to $310 \mathrm{~K}$ with a band gap width $\mathrm{E}_{\mathrm{g} \perp}=0.96 \mathrm{eV}$ for $\sigma_{\perp}$ and from 190 to $310 \mathrm{~K}$ with $\mathrm{E}_{\mathrm{g} / /}=1.1 \mathrm{eV}$ for $\sigma_{/ /}$. These values are in near agreement with the results obtained by Kashida et al. [12] and Nagat et al. [19]. Below $140 \mathrm{~K}$ the electrical conductivity is almost constant indicating the 
freezing of charge carriers. A previous work reported by Busch et al. [20] showed that the anisotropic factor of electrical conductivity $\left(\sigma_{\perp} / \sigma_{/ /}\right)$is independent of temperature, but in another previous investigation obtained by Schmid et al. [21], it was observed that $\left(\sigma_{\perp} / \sigma_{/ /}\right) \propto \exp \left(\mathrm{E}_{\mathrm{g}} / \mathrm{kT}\right)$. In the present work, it is clear that the temperature dependence of the $\left(\sigma_{\perp} / \sigma_{/ /}\right)$in TlS single crystal is in agreement with the latter investigation and is governed by the above relation (see Fig. (3)). This figure illustrates the variation of the anisotropic factor with temperature in the same investigated range, i.e. 100 to $360 \mathrm{~K}$, where this range includes four linear regions 140 to $180 \mathrm{~K}, 180$ to $210 \mathrm{~K}, 210$ to $260 \mathrm{~K}$ and 260 to $400 \mathrm{~K}$. The temperature coefficient of $\sigma_{\perp} / \sigma_{/ /}$in these four linear regions has the values $2.58 \times 10^{-4}, 1.42 \times 10^{-3}, 5.24 \times 10^{-4}$ and $7.43 \times 10^{-5}$, respectively. It is clear that the temperature dependence of $\left(\sigma_{\perp} / \sigma_{/ /}\right)$in the range 180 to $210 \mathrm{~K}$ is greater than those in the ranges 140 to $180 \mathrm{~K}, 210$ to $260 \mathrm{~K}$ and 260 to $400 \mathrm{~K}$. Figure (3) also suggests that $\sigma_{\perp}>\sigma_{/ /}$in the temperature range from 140 to $210 \mathrm{~K}$, but $\sigma_{\perp}<\sigma_{/ /}$in the range from 210 to $400 \mathrm{~K}$. So the electrical conductivity of TIS single crystal is highly anisotropic, which could be attributed partly or wholly to the interlayer macroscopic defects or planes of precipitates, where the low values of $\sigma_{/ /}$is due to the presence of the so-called "two dimensional defects" located between the layers that govern the carrier motion across the layers [21-23].

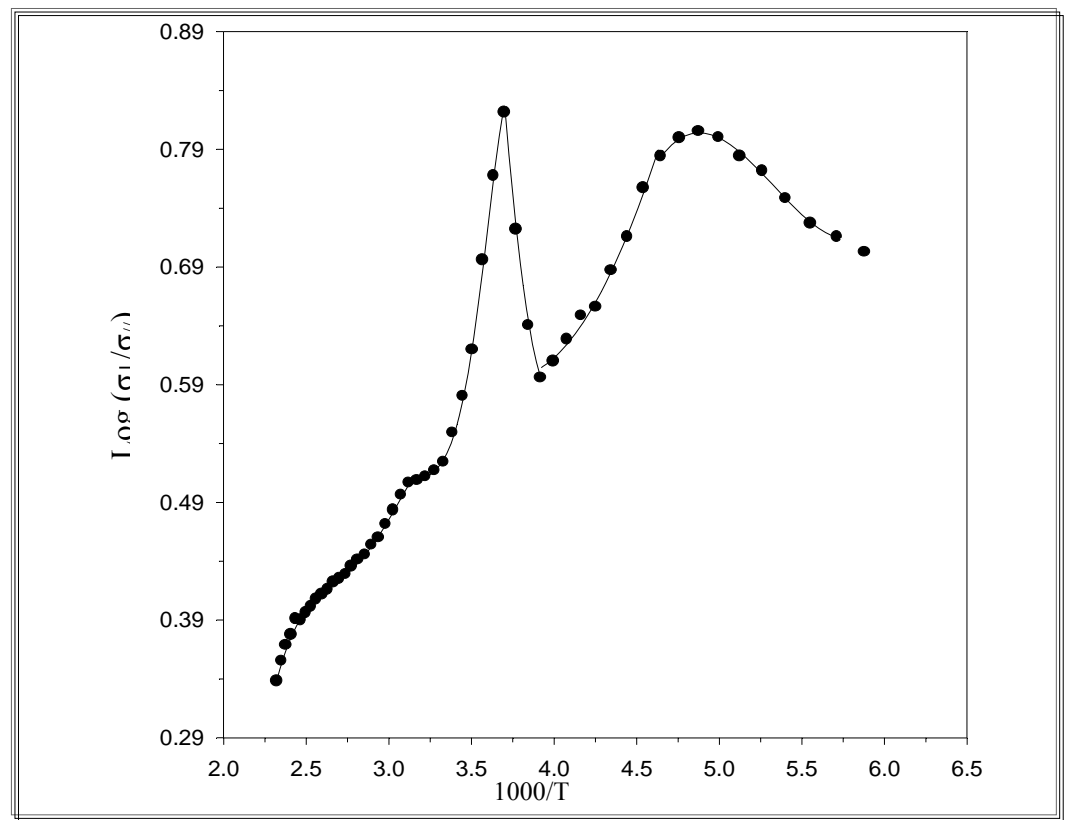

Fig. (3): Dependence of the anisotropic factor of electrical conductivity on temperatures for TIS single crystal. 
Dependences of the Hall coefficient on temperature for TIS single crystal is illustrated in Fig. (4). From this figure the positive sign of $R_{H}$ through the investigated temperature range indicates that TIS single crystal is a p-type semiconductors. The figure also suggests that, $R_{H}$ varies linearly with temperature in three regions extrinsic, transient and intrinsic. This shows that TIS specimen exhibit a considerable fall of $R_{H}$ at the temperatures referring to low temperature, but it falls rapidly in the transient region. In the intrinsic region, however $R_{H}$ falls more rapidly. At room temperature the Hall coefficient has a value of about $1.82 \times 10^{8} \mathrm{~cm}^{3} /{ }^{\circ} \mathrm{C}$ and the hole concentration " $\mathrm{p}$ " was calculated to be $3.44 \times 10^{10} \mathrm{~cm}^{-3}$.

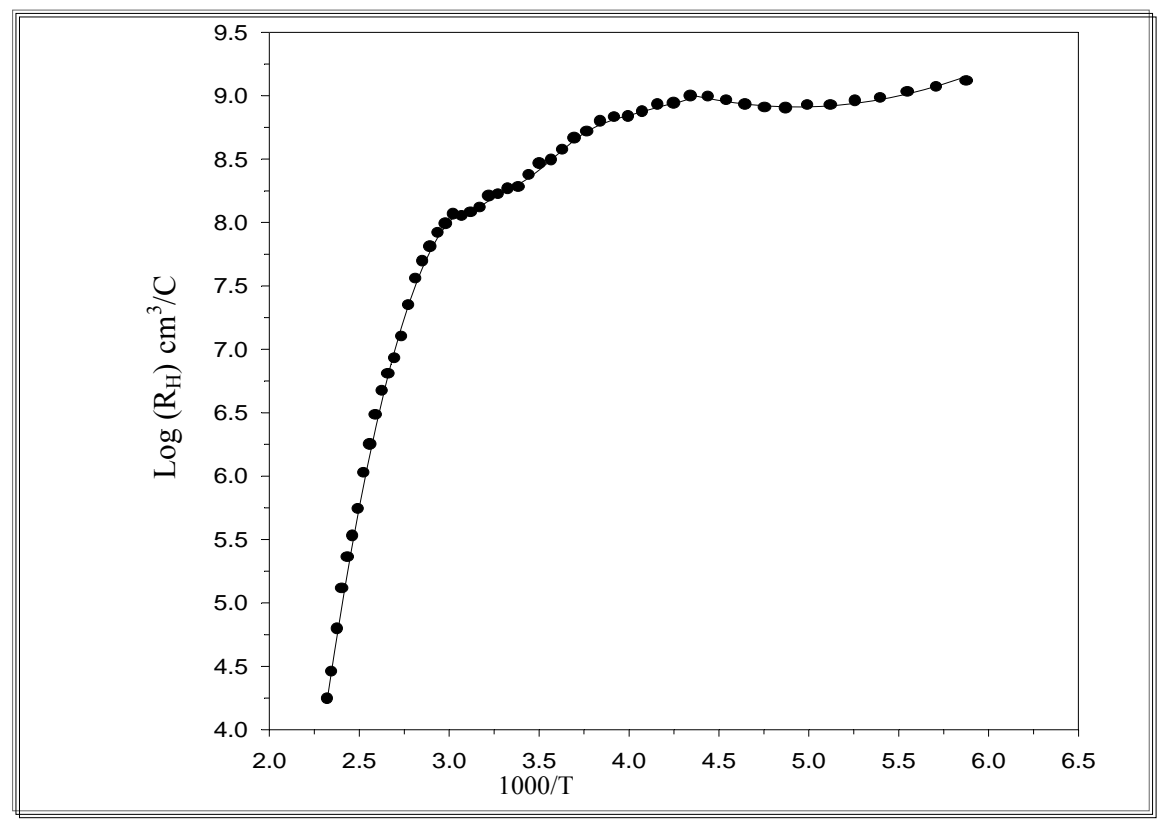

Fig. (4): The temperature dependence of Hall coefficient $\left(\mathrm{R}_{\mathrm{H}}\right)$ for TlS single crystal.

Relation between $\mathrm{R}_{\mathrm{H}} \mathrm{T}^{3 / 2}$ and 1000/T for T1S single crystal is depicted in Fig.(5) which shows that $R_{H} T^{3 / 2}$ increases linearly with increasing temperature. This linear increase is observed in three regions of the investigated temperature range (the same as those detected in the electrical conductivity measurements). With aid of this figure, one can compare and discuss the data calculated from this figure with that one given in the previous section. The intrinsic region is observed with forbidden gap width of $1.10 \mathrm{eV}$. The activation energy corresponding to the transient region has a value of about $0.13 \mathrm{eV}$ (activation energy has a value of about $0.17 \mathrm{eV}$ for $\sigma_{\perp}$ and $0.27 \mathrm{eV}$ for $\sigma_{/ /}$). The extrinsic region is observed with activation energy of $0.047 \mathrm{eV}\left(0.062 \mathrm{eV}\right.$ for $\sigma_{\perp}$ and $0.048 \mathrm{eV}$ for $\sigma_{/ /}$. 


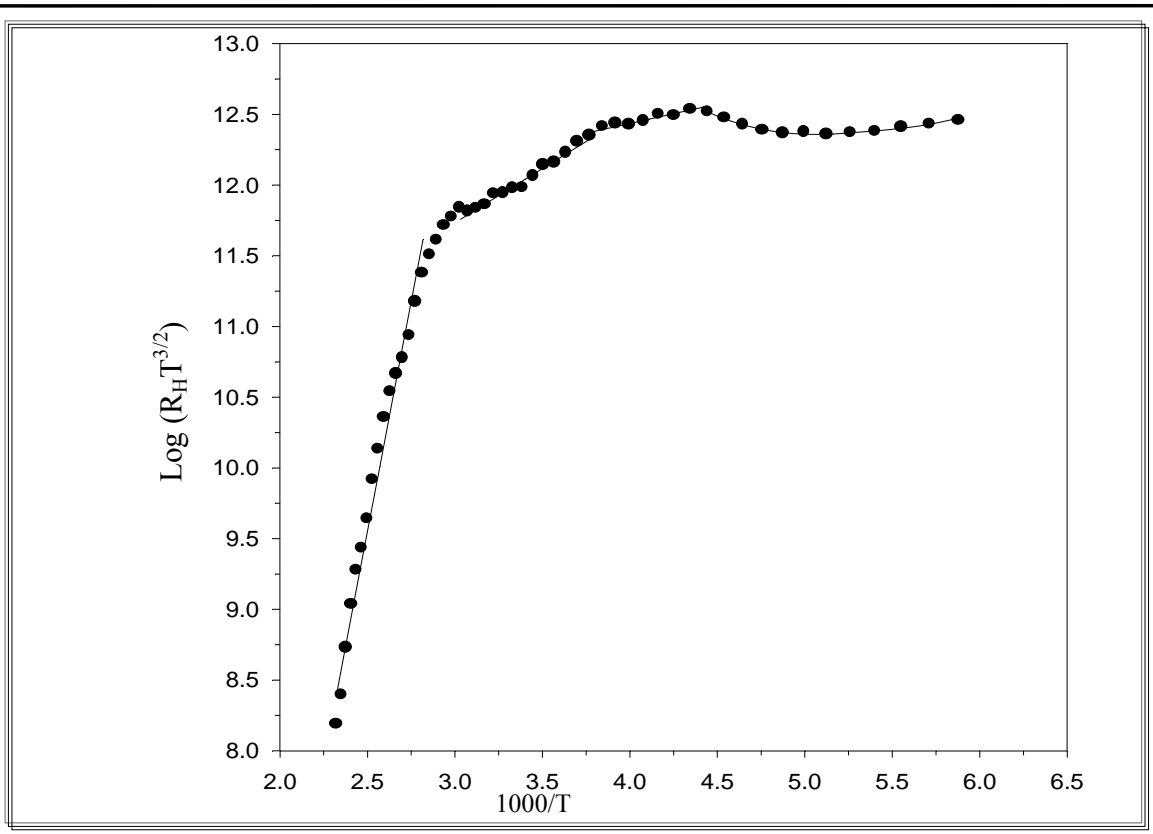

Fig. (5): Plot of $\left[R_{H} T^{3 / 2} \sim 1000 / T\right]$ for TIS single crystal.

Bube [24] concluded from the effect of scattering on the mobility of charge carries, that the mobility in materials with predominantly covalent bonding will usually be higher than the mobility in materials with predominantly ionic bonding. Bardeen and Shockley [25] reported the temperature dependence of the mobility as due only to interaction between charge carriers and acoustical vibrations in non-ionic crystals, for spherical equal-energy surfaces, to be in the form:

$$
\mu \alpha \mathrm{T}^{-3 / 2}
$$

The temperature dependence of the Hall mobility for T1S single crystals is illustrated in Fig. (6), which describes five linear behaviors of the Hall mobility with variation of temperature ( 170 to 210,255 to 290,290 to 335,335 to 390 and 390 to $430 \mathrm{~K}$ ): this figure shows that the linear dependence of $\log \mu$ on $\log \mathrm{T}$ in the low temperature range 170 to $210 \mathrm{~K}$ is observed with a slope of $1.69\left(\mu \alpha \mathrm{T}^{1.69}\right)$ for $\mu_{/ /}$measurement. Since this exponent is close to 1.5 one can say that the temperature dependence of the Hall mobility in this temperature interval may be due only to interaction between charge carriers and acoustical lattice vibrations [25]. However, in the high temperature regions there are four linear dependences of $\log \mu$ on $\log \mathrm{T}$ for both $\mu_{\perp}$ and $\mu_{/ / .}$. These are 255 to 290 $\mathrm{K}, 290$ to $335 \mathrm{~K}, 335$ to $390 \mathrm{~K}$ and 390 to $430 \mathrm{~K}$. For all the given high intervals of temperatures, the Hall mobility shows considerable deviation from the dependence given by Eq. 2 . 


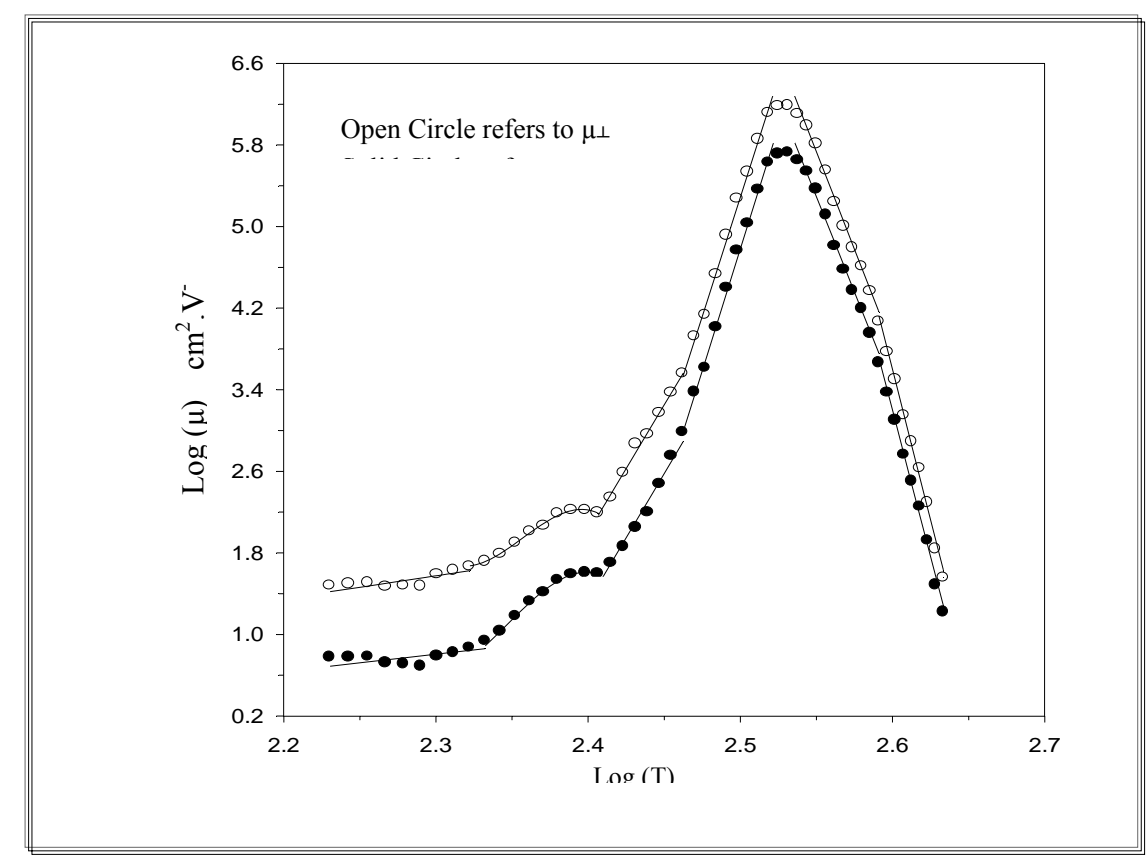

Fig. (6): Temperature dependence of Hall mobility for TIS single crystal.

Accordingly one can conclude the following: To our knowledge, there are little reported data in the literature on the electrical properties of TIS layered single crystal in the high temperature region. Anomalous behavior of the temperature dependence of Hall mobility similar to that observed in the present work has been reported by Nagat [26] in highly resistive thallium monosulphide single crystals and proposed to be due to a change in transport mechanism between localized states either within the energy gap or in the regions close to the band edges.

\subsection{Steady State Photoconductivity}

The total current (dark current + photocurrent) was measured at room temperature for different levels of illumination: $0.6 \mathrm{~F}, 1.2 \mathrm{~F}, 1.8 \mathrm{~F}, 2.4 \mathrm{~F}, 3 \mathrm{~F}$, $3.6 \mathrm{~F}$ and $4.2 \mathrm{~F}$, where $\mathrm{F}=1000$ Lux. The photocurrent was obtained by subtracting the dark current from the total current. The dependences of the dark and photocurrents on the applied voltage for thallium monosulfide single crystal is illustrated in Fig. (7). This figure shows that the dark current as well as the photocurrent linearly increases with increasing voltage in the whole investigated range of the applied voltage (i.e. 4 to $28 \mathrm{~V}$ ). The photosensitivity was determined for TIS single crystal using the relation $S=\left[\sigma_{\mathrm{ph}} / \sigma_{d}\right]$ for different 
levels of illumination to be $0.068,0.144,0.215,0.253,0.31,0.411$ and 0.48 for light intensities of $0.6 \mathrm{~F}, 1.2 \mathrm{~F}, 1.8 \mathrm{~F}, 2.4 \mathrm{~F}, 3 \mathrm{~F}, 3.6 \mathrm{~F}$ and $4.2 \mathrm{~F}$ respectively. The photocurrent was plotted as a function of the light intensity, Fig. (8). It is clear from this figure that the photocurrent linearly increases with increasing light intensity, which could be due to the generation of more free carriers. So the relation between the photocurrent and light intensity obeys the power law:

$$
\mathrm{I}_{\mathrm{ph}} \propto \mathrm{F}^{\gamma}
$$

where $\gamma$ is a constant, its value determines whether the recombination process is monomolecular or bimolecular. For $\gamma=0.5$, the recombination process is bimolecular, but if $\gamma=1$ the monomolecular process is dominated. If the value of $\gamma$ lies between 0.5 and 1 (sublinear), a continuous distribution of impurity states could be suggested [28]. In the present study, it is found that the value of $\gamma$ lies between 0.97 and 1.02, which indicates a monomolecular recombination process in TIS single crystals.

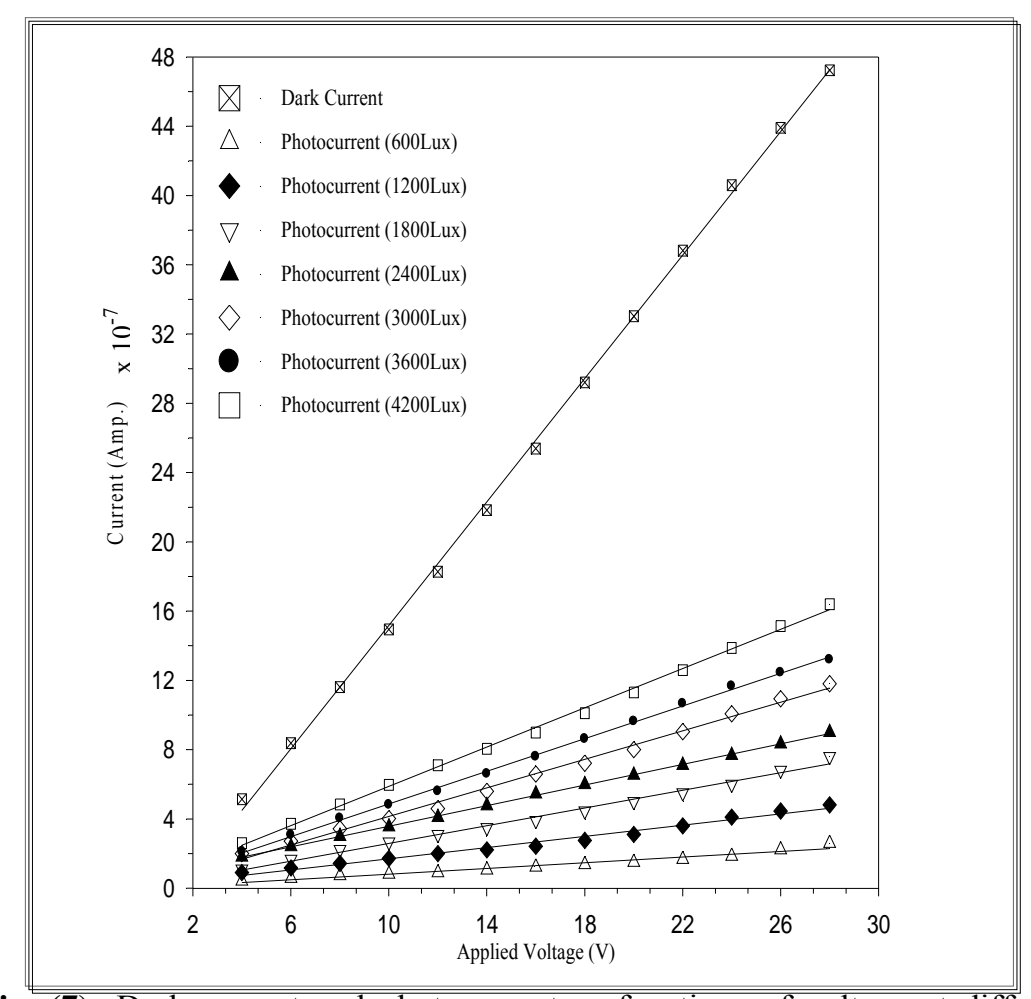

Fig. (7): Dark current and photocurrent as functions of voltage at different levels of illumination in TIS single crystal.

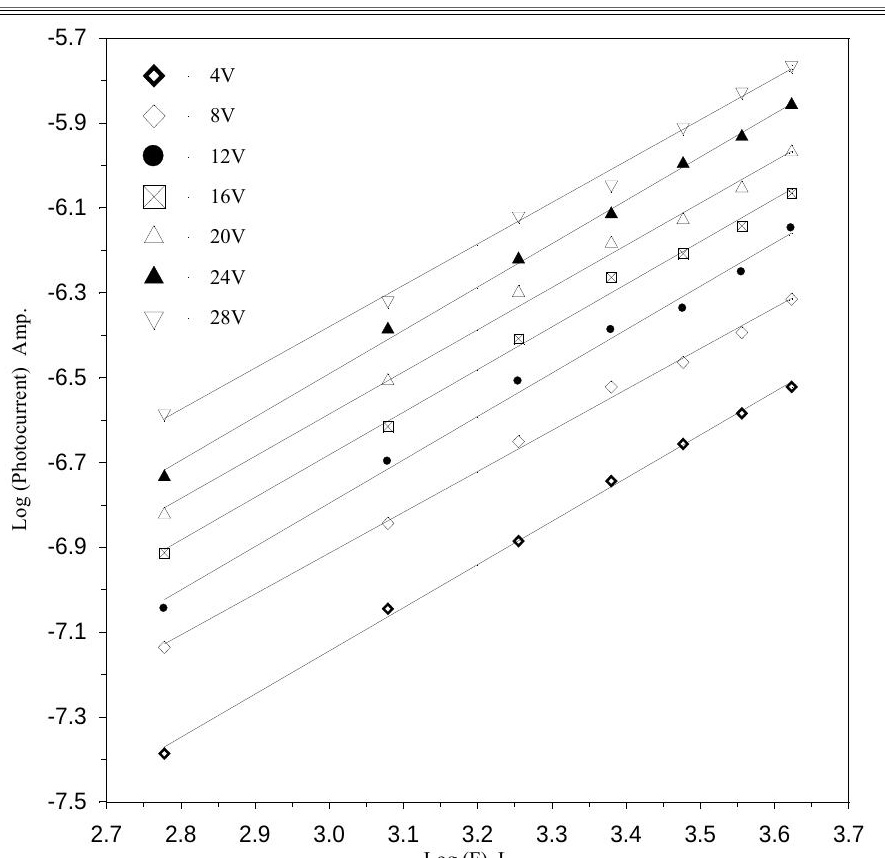


Fig. (8): Ligh intensity dependence of the photocurrent for TIS single crystal.

\section{Conclusion:}

The resultant crystal was characterized using XRD which showed that the obtained TIS compound is in single crystal form with tetragonal symmetry, space group: $14 / \mathrm{mcm}$ and lattice parameters: $\mathrm{a}=7.79 \AA$, $\mathrm{c}=6.79 \AA$ and $\mathrm{Z}=8$. The electrical condition was elucidated for the crystal, where it was found that $\sigma_{\perp}=8.9 \times 10^{-7}(\Omega . \mathrm{cm})^{-1}$ and $\sigma_{/}=1.39 \times 10^{-6}(\Omega . \mathrm{cm})^{-1}$. As a result of plotting $\left(\sigma_{\perp} / \sigma_{/ /}\right)$versus temperature, it was observed that the electrical conductivity in TIS single crystal is highly anisotropic especially in the temperature range $180 \mathrm{~K}$ to $210 \mathrm{~K}$. Photoconductivity measurements were studied in the applied voltage range $4 \mathrm{~V}$ to $28 \mathrm{~V}$ for different levels of illumination ranging from $0.6 \mathrm{~F}$ to $4.2 \mathrm{~F}(\mathrm{~F}=1000 \mathrm{Lux})$. The results showed that the photosensitivity of TIS single crystal varies from 0.068 to 0.48 when the light intensity changes from $0.6 \mathrm{~F}$ to $4.2 \mathrm{~F}$. It was found also that the recombination process in thallium monosulfide single crystal is monomolecular.

\section{Acknowledgement}

The authors are much indebted to Prof. Dr. A. E. Belal, Physics Department, Faculty of Science "Aswan", South Valley University, Egypt, for his help and encouragement. 


\section{References:}

1. K.A. Yee and A. Albright, J. Am. Chem. Soc. 113, 6474 (1991).

2. M.P. Hanias, A.N. Anagnostopoulos, K. Kambas and J. Spyridelis, Mat. Res. Bull. 27, 25 (1992).

3. N. Kalkan, J.A. Kalomiros, M.Hanias and A.N. Anagnostopoulos, Solid State Commun. 99, 375 (1996).

4. J.A. Kalomiros, N. Kalkan, M. Hanias, A.N. Anagnostopoulos and K. Kambas, Solid State Commun. 96, 601 (1995).

5. J.A.A. Ketelaar, W.H. t'Hart, M. Moerel, and D. Polder, Z. Kristallogr. 101, 396 (1939).

6. D. Muller and H. Hahn, Z. Anorg. Allg. Chem. 438, 258 (1978).

7. W. Henkel, H. D. Hochheimer, C. Carlone, A, Werner, S. Ves and H.G.V. Schnering, Phys. Rev. B 26, 3211 (1982).

8. S. Kashida and K. Nakamura, J. Solid State Chem., 110, 264 (1994).

9. A.M. Panich and S. Kashida, Physica B: Condensed Matter, 318, 217 (2002).

10. L. Porte and A. Tranquard, J. Solid State Chem. 35, 59 (1980).

11. J. M. Thomas and J. T. Michael, J. Chem. Soc. Faraday Trans. II, 70, 329 (1974).

12. S. Kashida, T. Saito, M. Mori, Y. Tezuka and S. Shin, J. Phys. Conden. Matt. 9 (46), 10271 (1997).

13. A. Mondal and P. Pramanik, Thin Solid Films, 110, 65 (1983).

14. S. Kashida, K. Nakamura and S. Katayama, Solid State Commun., 82, 127 (1992).

15. R.S. Itoga and C.R. Kannewurf, J. Phys. Chem. Solids 32, 1099 (1971).

16. K.R. Allakhverdiev, M.A. Nizametdinova, E. Yu. Salaev, R.M. Sardarly, N. Yu. Safarov, E.V. Vinogradov and G.N. Zhizhin, Solid State Commun. 36, 527 (1980).

17. I.M. Ashraf, H.A. Elshaikh, A.M. Nadr, J. Physics. Chem. B, 108, 31 (2004).

18. Hahn. Klinger, Z. Anorg. Allg. Chem., 260, 110 (1949).

19. A.T. Nagat, G.A. Gamal, Y.H. Gameel and N.N. Mohamed, Cryst. Res. Technol. 25, 4, K65 (1990).

20. G. Busch and O. Vogt, Helv. Phys. Acta, 33, 437 (1976).

21. M. Schmid and Ph.E. Mooser, Phys. Rev. B, 12 (1975).

22. M. Schluter, Nuovo Cimento B, 13, 313 (1973).

23. G.I. Belenkil and V.B. Stopachinshii, USP, Fiz. Nauk 140, 233 (1983).

24. R. Bube, "Photoconductivity of Solids", New York, Wiley (1960).

25. J. Bardeen and W. Shockley, Phys. Rev. 80, 72 (1950).

26. A.T. Nagat, J. Condens. Matter 1, 7921 (1989).

27. Albert Rose, "Concepts in Photoconductivity", New York: WileyInterscience, (1963). 